\title{
Norois
}

Environnement, aménagement, société

\section{Regards prospectifs spatialisés des entreprises bretonnes et ligériennes sur l'accessibilité ferroviaire nouvelle}

Prospective and spatialized views on the Bretagne-Pays de la Loire high-speed line by firms in Brittany and the Loire Valley

\section{Romaric Nègre}

\section{(2) OpenEdition}

\section{Journals}

\section{Édition électronique}

URL : http://journals.openedition.org/norois/6890

DOI : $10.4000 /$ norois. 6890

ISSN : $1760-8546$

\section{Éditeur}

Presses universitaires de Rennes

\section{Édition imprimée}

Date de publication : 5 novembre 2018

Pagination : 37-52

ISBN : 978-2-7535-7627-8

ISSN : 0029-182X

\section{Référence électronique}

Romaric Nègre, «Regards prospectifs spatialisés des entreprises bretonnes et ligériennes sur l'accessibilité ferroviaire nouvelle », Norois [En ligne], 248 | 2018, mis en ligne le 05 novembre 2020, consulté le 05 janvier 2021. URL : http://journals.openedition.org/norois/6890 ; DOI : https://doi.org/ 10.4000/norois. 6890 


Presses
Universitaires
de Rennes

\title{
Regards prospectifs spatialisés des entreprises bretonnes et ligériennes sur l'accessibilité ferroviaire nouvelle
}

\author{
Prospective and Spatialized Views on the Brittany-Pays de la Loire High Speed Line \\ by Firms in Brittany and the Loire Valley
}

\begin{abstract}
Romaric NÈGRE
Doctorant, UMR 6590 ESO, Université Rennes 2, Place du Recteur-Le-Moal, CS 24307, 35043 Rennes Cedex,

France. (romaric.negre@univ-rennes2.fr)
\end{abstract}

\begin{abstract}
Résumé : Il n'y a à ce jour de certitude scientifique sur la réalité ou sur l'ampleur éventuelle des effets territoriaux d'une nouvelle infrastructure de transport en général et d’une Ligne à Grande Vitesse en particulier. Les regards croisés de 179 dirigeants d'entreprise bretons et ligériens sur la modification des paramètres d'accessibilité ferroviaire dans le Grand Ouest entendent contribuer à éclairer un certain nombre de processus toujours mal cernés et de dynamiques interurbaines en cours ou à venir. En effet, les investissements privés de préfiguration et d'anticipation des changements d'accessibilité ferroviaire, liés à la mise en service de la LGV Bretagne-Pays de la Loire, ne sont pas homogènes au sein des territoires urbains du Grand Ouest. Une typologie des 13 agglomérations les plus directement concernées permet d'apprécier, toutes choses égales par ailleurs, le différentiel des incidences potentielles de la LGV Bretagne-Pays de la Loire sur les dynamiques des territoires urbains.
\end{abstract}

\begin{abstract}
To date, there is no scientific certainty regarding the existence and the potential extent of the territorial effects of new transport infrastructure in general, and of a high-speed rail line in particular. A comparative analysis of the views of 179 managers from Brittany and the Loire Valley on changes in rail-accessibility parameters in Western France seeks to shed light on certain processes that are still poorly understood, as well as on current and future interurban dynamics at play. In particular, private investments that foreshadowed and anticipated the commissioning of the Bretagne-Pays de la Loire high-speed rail line were not homogeneous across the main urban areas of Western France. A typology of the 13 most directly concerned cities makes it possible to evaluate, all else being equal, the differential in the potential impacts of the Bretagne-Pays de la Loire high-speed line on the dynamics of urban areas.
\end{abstract}

Mots clés : attractivité - train à grande vitesse - développement économique - prospective spatialisée

Keywords: attractiveness - high-speed rail-economical development-spatialized forward planning

\section{INTRODUCTION}

Depuis l'été 2017, la Ligne à Grande Vitesse Bretagne-Pays de la Loire (ci-après LGV BPL) a refondé les paramètres d'accessibilité ferroviaire du Grand Ouest français (tableau 1). Tête de proue des discours visant à réduire la périphéricité de la Bretagne dans un contexte de concurrence territoriale, l'infrastructure nouvelle véhicule des attentes fortes et incarne bien souvent des effets escomptés favorables (Bazin et al., 2006; Carrouet, 2013). 


\begin{tabular}{|c|c|c|c|c|c|c|c|c|c|c|c|c|c|}
\hline & \multicolumn{2}{|c|}{$\begin{array}{l}\text { Meilleur temps } \\
\text { de parcours TGV } \\
\text { avec Paris }\end{array}$} & \multicolumn{2}{|c|}{$\begin{array}{l}\text { Gain de temps } \\
\text { maximal }\end{array}$} & \multicolumn{2}{|c|}{$\begin{array}{c}\text { Temps de parcours } \\
\text { moyen TGV avec } \\
\text { Paris }\end{array}$} & \multicolumn{2}{|c|}{$\begin{array}{l}\text { Gain de temps } \\
\text { moyen }\end{array}$} & \multicolumn{2}{|c|}{$\begin{array}{l}\text { Fréquence } \\
\text { (nombre A/R } \\
\text { TGV/jour)* }\end{array}$} & \multicolumn{2}{|c|}{$\begin{array}{l}\text { Fréquence en } \\
\text { heure de pointe } \\
\text { (nombre A/R }^{\text {TGV/jour) }}{ }^{* *}\end{array}$} & \multirow{2}{*}{$\begin{array}{c}\text { Évolution } \\
\text { de la } \\
\text { fréquence/ } \\
\text { jour }\end{array}$} \\
\hline & $\begin{array}{c}\text { Avant } \\
\text { LGV }\end{array}$ & $\begin{array}{c}\text { Après } \\
\text { LGV }\end{array}$ & $\begin{array}{l}\text { Gain } \\
(\mathrm{mn})\end{array}$ & $\begin{array}{l}\text { Gain } \\
(\%)\end{array}$ & $\begin{array}{l}\text { Avant } \\
\text { LGV }\end{array}$ & $\begin{array}{c}\text { Après } \\
\text { LGV }\end{array}$ & $\begin{array}{l}\text { Gain } \\
(\mathrm{mn})\end{array}$ & $\begin{array}{c}\text { Gain } \\
(\%)\end{array}$ & $\begin{array}{c}\text { Avant } \\
\text { LGV }\end{array}$ & $\begin{array}{c}\text { Après } \\
\text { LGV }\end{array}$ & $\begin{array}{c}\text { Avant } \\
\text { LGV }\end{array}$ & $\begin{array}{c}\text { Après } \\
\text { LGV }\end{array}$ & \\
\hline Angers & $1 \mathrm{~h} 30$ & $1 \mathrm{~h} 24$ & 6 & 7 & $1 \mathrm{~h} 36$ & $1 \mathrm{~h} 31$ & 5 & 5 & 15,5 & 14,5 & 6 & 5,5 & $\Downarrow$ \\
\hline Brest & $4 \mathrm{hll}$ & $3 \mathrm{~h} 25$ & 46 & 18 & $4 \mathrm{~h} 27$ & $3 \mathrm{~h} 42$ & 45 & 17 & 8 & 10 & 1 & 2,5 & $\Uparrow$ \\
\hline Laval & $1 \mathrm{~h} 32$ & $1 \mathrm{~h} 11$ & 21 & 23 & $1 \mathrm{~h} 38$ & $1 \mathrm{~h} 25$ & 13 & 14 & 8 & 8 & 3,5 & 4 & $=$ \\
\hline Le Mans & $56 \mathrm{mn}$ & $54 \mathrm{mn}$ & 2 & 4 & $56 \mathrm{mn}$ & $55 \mathrm{mn}$ & 1 & 2 & 15,5 & 15,5 & 6,5 & 7 & $=$ \\
\hline Lorient & $3 \mathrm{~h} 38$ & $2 \mathrm{~h} 56$ & 42 & 19 & $3 \mathrm{~h} 48$ & $3 \mathrm{~h} 03$ & 45 & 20 & 7,5 & 9 & 2 & 3 & $\Uparrow$ \\
\hline Nantes & $2 \mathrm{~h} 05$ & $1 \mathrm{~h} 56$ & 9 & 7 & $2 \mathrm{~h} 17$ & $2 \mathrm{~h} 07$ & 7 & 5 & 19 & 19,5 & 6,5 & 7,5 & $=$ \\
\hline Quimper & $4 \mathrm{~h} 16$ & $3 \mathrm{~h} 31$ & 45 & 18 & $4 \mathrm{~h} 29$ & $3 \mathrm{~h} 42$ & 47 & 18 & 7 & 9 & 1,5 & 2,5 & $\Uparrow$ \\
\hline Rennes & $2 \mathrm{~h} 04$ & $1 \mathrm{~h} 25$ & 39 & 31 & $2 \mathrm{~h} 11$ & $1 \mathrm{~h} 39$ & 32 & 25 & 19,5 & 21 & 6,5 & 8,5 & $\Uparrow$ \\
\hline Sablé & $1 \mathrm{~h} 14$ & $1 \mathrm{~h} 05$ & 9 & 12 & $1 \mathrm{~h} 18$ & $1 \mathrm{~h} 11$ & 7 & 9 & 2,5 & 2,5 & 2 & 2 & $=$ \\
\hline St-Brieuc & $2 \mathrm{~h} 58$ & $2 \mathrm{~h} 13$ & 45 & 24 & $3 \mathrm{~h} 04$ & $2 \mathrm{~h} 22$ & 42 & 23 & 9 & 10,5 & 2,5 & 4 & $\Uparrow$ \\
\hline St-Malo & $2 \mathrm{~h} 56$ & $2 \mathrm{~h} 14$ & 42 & 24 & $3 \mathrm{~h} 07$ & $2 \mathrm{~h} 35$ & 32 & 17 & 3 & 3 & 0,5 & 1 & $=$ \\
\hline Vannes & $3 \mathrm{~h} 07$ & $2 \mathrm{~h} 29$ & 38 & 20 & $3 \mathrm{~h} 17$ & $2 \mathrm{~h} 35$ & 42 & 21 & 8 & 9,5 & 2,5 & 3,5 & $\Uparrow$ \\
\hline Vitré & $1 \mathrm{~h} 53$ & $1 \mathrm{~h} 31$ & 22 & 19 & $2 \mathrm{~h} 01$ & l h 45 & 16 & 14 & 2 & 2 & 1 & 2 & $=$ \\
\hline
\end{tabular}

Tableau 1 : Principales évolutions de l'offre ferroviaire avec la LGV BPL entre le Grand Ouest et Paris (Sources : SNCF - réalisation de l'auteur) Main changes in rail services following the commissioning of the Bretagne-Pays de la Loire HSL between Western France and Paris

* hors TGV « doublons»

** TGV avant 8 h 45 à l'arrivée et après 17 h 30 au départ

C'est que le projet de LGV BPL recoupe des questions vives touchant, notamment, à des enjeux importants sur les choix d'implantation des entreprises qui renvoient eux-mêmes à une possible reconfiguration du réseau urbain en termes d'attractivité et de fonctionnalité. Mais alors même que les LGV sont censées modifier l'aménagement du territoire, il est très difficile d'en mesurer les répercussions sur les marchés de l'emploi, la localisation des activités ou la productivité des entreprises. Il n'existe pas à ce jour de consensus académique sur la systématicité et l'ampleur des retombées économiques indirectes ${ }^{1}$ liées aux projets de transport en général et aux LGV en particulier (Bonnafous et Plassard, 1974; Vickerman, 1991 ; Offner, 1993; Bizeray et al., 1996 ; Lee, 2007 ; Mignerey, 2012; Albalate et Bel, 2012). Synthétisant l'état actuel de la connaissance scientifique sur le sujet, la Commission Quinet (CGSP, 2013 , p. 27) souligne ainsi l'absence «de résultats suffisamment robustes pour conclure que les investissements en infrastructures ont un impact positif

1. Il convient de parler de «wider economic impacts » en reprenant la terminologie anglo-saxonne (Vickerman et Ulied, 2012) ou de « retombées économiques indirectes » dans le cas français. sur la croissance, en dehors de celui correspondant aux surplus du calcul économique ».

Certes, il existe bien dans les bilans $\mathrm{LOTI}^{2}$ de nombreux effets territoriaux attendus ex ante, mais les études ex post sont plus que nuancées. Le bilan LOTI de la LGV Atlantique indique par exemple que l'impact sur l'aménagement du territoire et la dynamique urbaine est peu perceptible, même s'il ne peut être négligé. De manière similaire, le bilan LOTI de la LGV Nord établit que l'impact sur l'investissement et sur l'emploi local est particulièrement difficile à évaluer. Enfin, les comparaisons des performances économiques des régions françaises (Crozet, 2013) et principales métropoles (Mattmuller, 2016), en fonction du calendrier d'introduction des LGV, montrent des résultats contrastés.

En somme, si l'état de l'art n'autorise pas de conclusions définitives et générales sur les changements de localisation des activités, la possible recomposition de l'attractivité et la dynamisation des

2. Documents règlementaires d'évaluation environnementale et socio-économique produits 3 à 5 ans après la mise en service des grandes infrastructures de transport. Il incombe ainsi au maître d'ouvrage d'analyser et d'expliquer les écarts entre l'évaluation économique et sociale du projet établie à l'issue de l'enquête publique (situation initiale ou ex ante) et les observations réelles après la mise en service (situation ex post). 
territoires concernés par l'implantation d'une ligne à grande vitesse (Offner, 1993; Klein et Million, 2007 ; Ureña et al., 2009; Bazin et al., 2010; Delaplace et al., 2013), il soulève par là même toute l'acuité de ces problématiques pour les chercheurs et la société civile. Au vu de l'imposante envergure budgétaire des projets d'infrastructure ferroviaire à grande vitesse, il s'agit d'enjeux trop importants pour qu'on ne cherche pas à s'en saisir.

S’inscrivant dans cet élan, la présente contribution entend éclairer des processus toujours mal cernés à travers un positionnement scientifique singulier fondé sur le regard des chefs d'entreprise. La plupart des études de cas entourant l'arrivée de la grande vitesse ferroviaire n'apportent qu'un regard à la marge sur les stratégies managériales privées d'anticipation et d'appropriation, privilégiant à l'inverse le discours des acteurs institutionnels (Menerault, 2000; Blanquart et Delaplace, 2009; Carrouet, 2013).

Il faut dire que les quelques travaux publiés sur les comportements des entreprises en amont de la mise en service d'une LGV concluent à la quasi absence d'anticipation de ces acteurs. Un rapport de recherche pour le conseil régional de ChampagneArdenne, dressant une évaluation prospective des impacts de la LGV est-européenne sur Reims et sa région un an avant l'exploitation de l'infrastructure (Bazin et al., 2006), observe une faible adhésion générale et des anticipations limitées de la part des entreprises champardennaises. Des années auparavant, l'arrivée de la LGV Atlantique avait fédéré l'Observatoire économique et statistique des transports (OEST), la DATAR et la Direction des transports terrestres, dans la conduite d'un panorama approfondi des dynamiques territoriales du Mans (Chevalier, 1990; Gac, 1990) et de Nantes (Paumier, 1990). Leurs conclusions respectives sont puissamment convergentes : " le résultat le plus marquant de cette série d'entretiens est l'absence quasi-totale d'anticipation de la nouvelle infrastructure pour l'activité de l'entreprise [...]. Sur le plan de l'organisation des marchés, de la stratégie commerciale des entreprises, des relocalisations possibles d'antennes ou de filiales, les entrepreneurs ne perçoivent pas l'enjeu que pourrait représenter le TGV » (Paumier, 1990, p. 120).

Partant d'une époque qui n'est plus guère comparable aux années des premiers investissements dans la grande vitesse ferroviaire, la perspective entrepre- neuriale donnée à ce travail consiste donc à évaluer la sensibilité des entreprises privées ${ }^{3}$ locales à la modification des paramètres d'accessibilité ferroviaire dans le Grand Ouest. L'aire d'étude couvre ainsi un ensemble de 13 agglomérations bretonnes et ligériennes de différentes tailles (Angers, Brest, Laval, Le Mans, Lorient, Nantes, Quimper, Rennes, Sablé-sur-Sarthe, Saint-Brieuc, Saint-Malo, Vannes et Vitré), comme autant de contextes territoriaux spécifiques. En examinant tous les niveaux de la hiérarchie urbaine, ce travail issu d'une thèse (Nègre, 2018) se distingue de la majorité des publications ayant privilégié l'analyse des effets entre la régioncapitale et les principales métropoles régionales (Bavoux et Piquant, 2000).

L'objectif de l'article est ainsi de cerner les incidences potentielles de la LGV BPL sur l'organisation des entreprises dans le Grand Ouest à travers la perception de leur dirigeant. Il s'agit tout d'abord de montrer comment les acteurs stratégiques des territoires que sont les entreprises, appréhendent et s'emparent des changements d'accessibilité ferroviaire liés à la mise en service de la LGV BPL, si tant est qu'elles démontrent des signes en ce sens. Car il y a en réalité un double champ de la sensibilité : celui du « sensible » et de l'émotivité d'une part, ne renfermant pas automatiquement de visée stratégique affirmée, et celui du processus d'appropriation pouvant conduire à l'action stratégique d'anticipation.

Après avoir présenté le matériau qualitatif constituant le socle de ce travail, nous tâchons de proposer une analyse réflexive de la capacité des entreprises à la préfiguration des changements d'accessibilité ferroviaire. Le second volet de cette contribution illustre ensuite le passage du discours des dirigeants d'entreprise aux dynamiques urbaines, en esquissant une typologie territoriale de la sensibilité entrepreneuriale à l'accessibilité ferroviaire nouvelle.

\footnotetext{
3. L'exclusion des établissements publics résulte d'une délimitation volontaire, étant admis que le statut public rend bien souvent compte de considérations institutionnelles et administratives qui, d'une part atténuent de facto l'autonomie stratégique des entités régionales, et d'autre part ne répondent pas aux mêmes stratégies d'implantation et logiques de marché que les établissements privés.
} 


\section{DU DISCOURS DES DIRIGEANTS D'ENTREPRISE À LA DÉFINITION DES SENSIBILITÉS TERRITORIALES}

\section{L'entrée par la firme : regards croisés de 179 dirigeants d'entreprise bretons et ligériens}

Paradoxalement, peu nombreux sont les travaux analysant le lien entre infrastructures de transport et développement économique à travers le prisme des pratiques et stratégies d'entreprise (Giuliani, 2007; Bouba-Olga et al., 2012; Blanquart et al., 2013). Régler la focale sur la firme permet pourtant l'identification des liens relationnels inscrits dans l'espace, ainsi que l'étude de certaines composantes organisationnelles partagées avec le territoire, comme l'accès à la ressource humaine ou matérielle (Martin et Sunley, 2007).

Appréhender le regard du chef d'entreprise sur l'accessibilité ferroviaire, c'est foncièrement partir de deux questionnements en apparence triviaux : pourquoi les professionnels se déplacent-ils, et sur quoi « gagne »-t-on du temps? La question de l'évolution des pratiques de déplacements ferroviaires longue distance est surement la plus documentée dans la littérature s'étant intéressée à l'incidence des LGV
(Klein, 1997). Mais au-delà du vaste champ de la mobilité et de l'usage, l'appréhension pertinente de la modification des accessibilités ferroviaires nécessite de couvrir des thèmes relevant de la dimension stratégique des organisations, de l'image, voire du non-usage (figure 1). Les localisations, étant entendues comme des dimensions constitutives de l'activité (Perrat, 2000), sont transversales à l'ensemble des axes thématiques car « organisation, usage du temps et de l'espace sont indissociables » (Klein, 2005, p. 29).

\section{Méthodologie de l'enquête}

Afin de répondre à notre problématique, qui porte sur les potentielles incidences territoriales de la LGV BPL révélées par la sensibilité ex ante des entreprises, la rencontre avec des acteurs productifs du Grand Ouest s'est imposée. Un guide, composé d'un registre de questions semi-directives articulées en 4 thèmes, a servi de fil conducteur aux échanges (figure 1). Au sein de chacun de ces thèmes, la discussion a systématiquement été divisée en deux temps. Celui de la situation actuelle, à travers la caractérisation des déplacements et l'exposé de l'organisation spatio-productive, puis celui

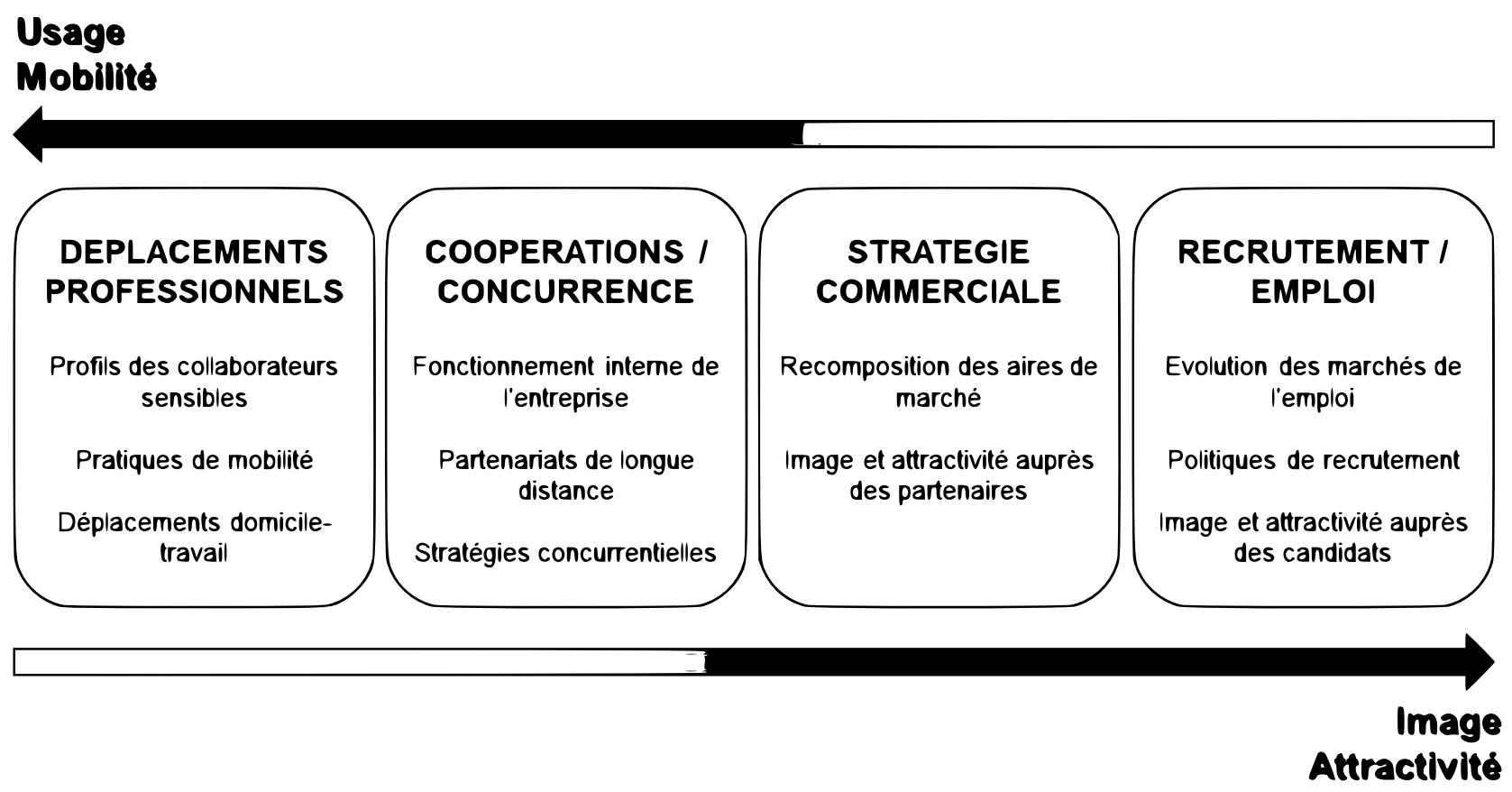

Figure 1 : Thèmes portés à discussion avec les dirigeants d'entreprise Topics selected for discussion with business leaders 
de la situation projetée après la mise en service de la LGV BPL.

En outre, l'échantillonnage en vue de l'enquête de terrain procède d'un certain nombre de choix raisonnés qu'il convient de présenter brièvement. Si la sélection des entreprises ne provient pas d'une méthode probabiliste qui vaudrait représentativité statistique, il s'avère nécessaire de veiller à ce que l'échantillon empirique soit néanmoins explicatif de certains éléments des trajectoires territoriales.

La procédure de sélection trouve son origine dans les bases de données Diane et Astrée. Face à la donnée brute retraçant l'ensemble des entités françaises en activité, un premier filtre géographique a été appliqué. Ainsi, nous n'avons retenu dans la base principale que les établissements localisés au sein des 13 aires urbaines du Grand Ouest qui constituent notre périmètre d'étude. Une deuxième sélection a ensuite été opérée par le biais des sphères et domaines d'activité principale. Ont été écartées les activités de la sphère présentielle afin que soient privilégiées les rencontres avec des acteurs de la sphère productive, en fonction de leur ordre de grandeur dans les portefeuilles économiques locaux. La présence de liens entre établissements parmi les 13 aires urbaines et la région parisienne figure également parmi les indicateurs.

Le processus d'identification des entreprises a en parallèle été enrichi par d'autres sources d'information, issues notamment de données locales (fichiers CCI, annuaires économiques...), de la littérature non académique ou des médias spécialisés. Nous avons confronté la liste précédemment constituée aux connaissances de divers partenaires sur le tissu local d'entreprises à travers une série de rencontres dans les territoires. En définitive, l'échantillon d'entreprises final s'établit à 634 entreprises.

Au terme de dix mois de rencontres en 2016, 179 responsables d'entreprise ${ }^{4}$ ont pu être sollicités au sein des 13 entités territoriales inscrites au périmètre de l'étude (figure 2 - planche IX). Pour donner un point de repère bien plus qu'en considérant les chiffres exploitables, cela équivaut à environ 34000 emplois salariés, soit $3 \%$ de l'emploi total des 13 agglomérations du Grand Ouest ${ }^{5}$ (tableaux 2). À

4. Dans $92 \%$ des cas, il s'agit de la personne à la tête de l'entreprise ou de l'établissement (président, directeur général, directeur de site, gérant...). 5. N'ont été retenus que les effectifs de l'entité sur le territoire dans lequel l'entretien a eu lieu, et ce même si d'autres établissements existent dans un ou plusieurs autre(s) territoire(s) de l'étude ou si l'interlocuteur a une défaut d'une parfaite représentation statistique, ces échanges entendent décrire un large spectre des incidences de la LGV BPL sur l'organisation interurbaine des principales activités du territoire. La liste couvre ainsi une variété de structures différentes par leur taille ${ }^{6}$, leur secteur d'activité ${ }^{7}$, leur étendue de marché, leur ancienneté, leur organisation interne $^{8}$, etc. Cette diversité souhaitée n'est autre que l'application pratique de notre questionnement de recherche principal, à savoir l'identification des paramètres discriminants et faits stylisés spatialisés de la sensibilité des entreprises privées à l'accessibilité ferroviaire accrue.

À noter que si l'échantillon à l'origine des demandes d'entretien se voulait grossièrement représentatif des composantes économiques des territoires, les caractéristiques des entreprises ayant bien voulu être sollicitées divergent logiquement de la représentativité idéale ${ }^{9}$. Préalablement à l'analyse des sensibilités associées à chacun des 13 territoires, il convient dès lors de présenter sommairement quelques indicateurs synthétiques de la composition économique des entités géographiques et des entreprises du panel (tableaux 2).

responsabilité au-delà du territoire enquêté. A titre d'exemple, bien que nous ayons rencontré le directeur régional du groupe Orange à Rennes, nous retenons comme effectif les 5000 collaborateurs de l'aire urbaine rennaise et non les 8500 salariés du Groupe en région Bretagne.

6. Comme nous l'indiquions précédemment, les éventuels effectifs des sociétés hors du territoire dans lequel l'entretien est conduit ne sont pas intégrés au calcul. Il s'agit donc d'estimer la taille des établissements locaux et non celle des entreprises. Avec une valeur médiane de 45, les structures du corpus apparaissent de taille moyenne. Plus de la moitié d'entre elles comptent de 10 à 249 employés. Sept établissements possèdent toutefois plus de 1000 salariés, le plus grand atteignant même 5000 collaborateurs

7. Globalement, les entreprises rencontrées exercent à $25 \%$ dans le secteur industriel, à $35 \%$ dans le numérique (conseil technologique, édition, programmation, télécommunications...), à près de $30 \%$ dans les services aux entreprises hors numérique et banques (activités juridiques et comptables, conseil en gestion, ingénierie et études, publicité...) et près de $10 \%$ dans la bancassurance.

8. $78 \%$ des structures rencontrées (soit 135 entreprises) ont leur siège social ou leur établissement principal dans le Grand Ouest et $22 \%$ (soit 38 entreprises) sont des établissements secondaires ou des filiales. $65 \%$ des sièges et établissements principaux sont mono-établissement, $24 \%$ ont un établissement secondaire en Île-de-France et $26 \%$ en possèdent un dans l'un des autres territoires du Grand Ouest inscrit au périmètre d'étude. $91 \%$ des établissements secondaires ou filiales ont leur tête de groupe nationale en région francilienne.

9. Pour autant, nous ne voulons pas affirmer ici que l'absence de concrétisation de l'entretien est une forme d'insensibilité à l'arrivée de la grande vitesse ferroviaire en Bretagne. Les raisons sont autrement plus complexes. Le nombre d'entretiens est avant tout la résultante de l'acceptation de la rencontre par les chefs d'entreprise. Nous avons en outre considéré que Laval et Rennes étaient les deux bénéficiaires directes nouvelles de l'arrivée d'une LGV dans le Grand Ouest. En fin de compte, ces deux territoires présentent à eux deux plus de la moitié des entretiens. Laval a également fait valoir un taux de concrétisation des entretiens plus important que les autres territoires $(40 \%$, soit 28 entreprises rencontrées pour 70 sollicitées). 


\begin{tabular}{|l|c|c|c|c|}
\hline Aires urbaines & Emploi total en 201 l & $\begin{array}{c}\text { Taux d'évolution de l'em- } \\
\text { ploi entre 2006 et 201 l }\end{array}$ & $\begin{array}{c}\text { Part des cadres des fonc- } \\
\text { tions métropolitaines dans } \\
\text { l'emploi total en 2011 }\end{array}$ & $\begin{array}{c}\text { Taux d'évolution des } \\
\text { CFM }^{*} \text { entre 2006 et 201 1 }\end{array}$ \\
\hline Angers & 167939 & 2,24 & $7,3 \%$ & 5,58 \\
\hline Brest & 130674 & 2,97 & $8,1 \%$ & 16,25 \\
\hline Laval & 57291 & 0,45 & $7,2 \%$ & 23,36 \\
\hline Le Mans & 139779 & $-1,04$ & $7,0 \%$ & 5,36 \\
\hline Lorient & 83617 & 1,78 & $6,1 \%$ & 15,86 \\
\hline Nantes & 402164 & 7,28 & $11,6 \%$ & 7,31 \\
\hline Quimper & 59295 & 0,68 & $6,9 \%$ & 17,49 \\
\hline Rennes & 309353 & 5,58 & $11,4 \%$ & $-1,90$ \\
\hline Sablé s/Sarthe & 16048 & 3,40 & $3,1 \%$ & 15,76 \\
\hline Saint-Brieuc & 72867 & 2,54 & $6,1 \%$ & 17,15 \\
\hline Saint-Malo & 31661 & 0,36 & $6,1 \%$ & 6,68 \\
\hline Vannes & 65978 & 4,34 & $7,2 \%$ & 27,08 \\
\hline Vitré & 15136 & $-1,58$ & $5,4 \%$ & \\
\hline
\end{tabular}

Tableau 2A : Indicateurs synthétiques de l'emploi dans les aires urbaines du panel Composite indicators of employment in the urban areas studied

* Cadres des fonctions métropolitaines

\begin{tabular}{|c|c|c|c|c|c|c|c|c|}
\hline $\begin{array}{c}\text { Aires } \\
\text { urbaines }\end{array}$ & $\begin{array}{c}\text { Emplois } \\
\text { dans la } \\
\text { production } \\
\text { manufactu- } \\
\text { rière (2015) }\end{array}$ & $\begin{array}{c}\text { Emplois } \\
\text { dans le panel } \\
\text { d'entreprises } \\
(2017)\end{array}$ & $\begin{array}{c}\text { Production } \\
\text { et conseil } \\
\text { numérique } \\
(2015)\end{array}$ & $\begin{array}{c}\text { Dans le } \\
\text { panel } \\
\text { d'entreprises } \\
(2017)\end{array}$ & $\begin{array}{c}\text { Services spé- } \\
\text { cialisés aux } \\
\text { entreprises } \\
(2015)\end{array}$ & $\begin{array}{c}\text { Dans le } \\
\text { panel } \\
\text { d'entreprises } \\
(2017)\end{array}$ & $\begin{array}{c}\text { Banque et } \\
\text { assurance } \\
(2015)\end{array}$ & $\begin{array}{c}\text { Dans le } \\
\text { panel } \\
\text { d'entreprises } \\
(2017)\end{array}$ \\
\hline Angers & 25058 & 22 & 2693 & 194 & 3016 & 280 & 6057 & - \\
\hline Brest & 14196 & 206 & 1798 & 27 & 3137 & 108 & 4806 & 277 \\
\hline Laval & 9715 & 1893 & 869 & 113 & 705 & 245 & 2263 & 693 \\
\hline Le Mans & 21832 & 319 & 1523 & 23 & 1754 & 42 & 7619 & 3665 \\
\hline Lorient & 14495 & 2486 & 980 & - & 1435 & 11 & 1445 & 36 \\
\hline Nantes & 56046 & 26 & 22099 & 361 & 16902 & 5 & 17958 & - \\
\hline Quimper & 9389 & 773 & 769 & 131 & 926 & 45 & 2866 & 291 \\
\hline Rennes & 45677 & 3513 & 16334 & 7138 & 10992 & 395 & 9973 & 1959 \\
\hline Sablé & 6393 & 2444 & 22 & - & 82 & 6 & 140 & - \\
\hline St-Brieuc & 10770 & 512 & 583 & 81 & 1342 & 138 & 2348 & - \\
\hline St-Malo & 4706 & 2143 & 321 & - & 412 & 9 & 569 & - \\
\hline Vannes & 9064 & 805 & 1221 & 236 & 1079 & 1 & 2253 & 20 \\
\hline Vitré & 5753 & 1115 & 15 & - & 253 & 0 & 266 \\
\hline
\end{tabular}

Tableau 2B : Indicateurs sectoriels de l'emploi dans les aires urbaines et le panel d'entreprises (sources : CLAP 2015 et RGP Insee, chiffres issus de l'enquête, de l'auteur)

Sectoral indicators of employment in the urban areas and firms studied

Notes : Certaines entreprises classées dans une catégorie « autres secteurs » ne sont pas prises en compte ici. Leur intégration dans l'échantillon étant souvent due à leur qualité de «porte-drapeaux» du territoire, elles présentent fréquemment un nombre de salariés conséquent. 


\section{Les grands déterminants de la sensibilité des entreprises à la LGV BPL}

Les déterminants de la prédisposition différenciée des entreprises à "réfléchir la LGV » sont multiples (Klein, 2001). Nous en présentons ici brièvement trois parmi les principaux. Premièrement, l'étendue spatiale du marché met en évidence, de façon somme toute logique, que la grande vitesse ferroviaire n'a éventuellement des répercussions que sur les entreprises dont le marché est national voire international (Buisson, 1986; FacchinettiMannone, 1995; Ollivro, 1997; Bazin et al., 2006). Néanmoins, un marché local n'est pas nécessairement incompatible avec des déplacements à motif d'interlocution externe (rencontres événementielles ou institutionnelles notamment) ou des déplacements à motif informationnel internes. Ces derniers sont particulièrement révélateurs de la mobilité des professionnels des banques fédéralistes régionales. Malgré une aire de clientèle locale, les déplacements vers la région francilienne sont fréquents et stratégiques. Au sein des groupes bancaires fédéralistes, la dispersion spatiale du savoir et la valeur assurantielle associée à l'échange interne, imposent un besoin croissant de relations de coopération "face-à-face » dans certains segments complexes tels la conjoncture ou les risques.

Deuxièmement, l'existence de liens interterritoriaux entre siège et établissements régionaux influe indéniablement sur la sensibilité stratégique des structures privées. Sur ce point, les résultats empiriques concluent à un usage professionnel relativement différencié des modes aérien, ferroviaire ou routier entre usagers franciliens et voyageurs en région (Klein, 1997). En effet, les établissements régionaux de nombreuses entités de service aux entreprises, qui fonctionnent généralement comme un centre de profit autonome du siège francilien, ne disposent pas de l'envergure financière de leur tête de groupe. Aussi ont-ils des pratiques de déplacement plus contraintes par des exigences de coût.

Enfin, s'il était a priori envisagé que le degré de sensibilité diffère selon le secteur d'activité (Facchinetti-Mannone, 1995; Dornbusch, 1997; Bazin et al., 2006), les entretiens menés tendent à tempérer cette assertion. Il semblerait en effet que le secteur ne soit pas intrinsèquement attaché à un volume de mobilité longue distance spécifique mais à des caractéristiques propres. Par exemple, à taille comparable et bien que situé en périphérie, un grand équipementier automobile présente aisément un volume de déplacements en TGV identique à une entreprise de conseil technologique. Seulement ses déplacements, orientés vers les clients et partenaires industriels, sont avant tout à destination de l'Est de la France et l'Ouest de l'Allemagne, alors que les entreprises du numérique ont une clientèle extra-locale essentiellement francilienne.

\section{La confortation des schémas organisationnels et spatiaux existants}

À partir des précédentes observations de la sensibilité des entreprises, il s'agit à présent d'amorcer le passage délicat à la prospective de potentielles nouvelles configurations économiques des territoires. Au-delà de la firme ou de la filière, la sensibilité des entreprises privées est en partie " supradéterminée » par l'appartenance territoriale et le contexte macroéconomique. S'il est relativement admis que la taille de l'agglomération ne suffit à elleseule à expliquer les effets d'une LGV, la littérature confère néanmoins aux territoires des potentialités de prédisposition, en fonction notamment de leur portefeuille d'activités. Les travaux de MérenneSchoumaker (1996 et 2011), les développements de l'École dite de la proximité (Torre et Rallet, 2005) et la « Nouvelle Économie Géographique » relative aux transports (Combes et al., 2006; Lafourcade et Thisse, 2009) ont contribué à faire émerger les cadres explicatifs récents des choix de localisation des firmes. Ainsi, les dotations économiques et infrastructurelles initiales, les trajectoires en cours, l'attractivité productive et résidentielle, l'effort institutionnel local d'accompagnement de l'« événement LGV », sont autant de composantes du référentiel spatial de la sensibilité des entreprises.

Délier le développement des entreprises et les dynamiques des territoires n'est donc pas chose souhaitée dans ce travail, et ce pour deux raisons principales. La première résulte de la difficulté, voire de l'impossibilité (Frémont, 2014), de distinguer dans les discours des dirigeants, l'incidence directe de l'accessibilité accrue et celle imputable aux contextes interne ou externe à l'entreprise. La seconde tient au caractère permissif des infrastructures de transports (Bérion, 1998), qui ne sont que 
des supports à l'exercice de l'activité et des outils à la rencontre physique entre individus. Isoler la contribution nette de la LGV BPL pour un chef d'entreprise n'a aucun sens tant l'infrastructure entre en congruence avec les fondements et la réalisation même de ses activités.

D'autant plus que la LGV BPL conforte plus qu'elle ne bouleverse les choix stratégiques préalables inhérents à l'organisation de la production des biens et services. Autrement dit, rares sont les entreprises qui envisagent des modifications significatives de leurs stratégies en capitalisant sur l'accessibilité ferroviaire accrue ${ }^{10}$. L'expression des dirigeants s'en ressent : "c'est un peu difficile d'évaluer l'effet TGV, ce n'est pas encore concret »; " pour des réorientations stratégiques c'est trop tôt et encore flou » indiquent-ils. Cette confortation générale des paramètres organisationnels et spatiaux actuels traduit, pour l'heure, le caractère peu discriminant des apports de la LGV au-delà des considérations de confort et de flexibilité des déplacements permises par un « effet d'aubaine». Elle tient aussi parfois d'une capacité de projection limitée des dirigeants, pour qui la temporalité de l'action est courte et seule l'expérience du déplacement se révèle être un outil de projection des stratégies.

Le présent travail de prospective se démarque donc à la fois des analyses exclusivement a posteriori et des nombreux travaux relevant de la comparaison (souvent difficile et imparfaite, à l'image des bilans LOTI) de deux situations temporellement disjointes. Le regard des entreprises projeté sur les territoires (Martin et Sunley, 2007; Bouba-Olga et al., 2012) met en lumière les interrelations économiques, en cours et potentiellement à venir, au sein des systèmes urbains du Grand Ouest.

\section{TYPOLOGIE TERRITORIALE DE LA SENSIBILITÉ À L'ACCESSIBILITÉ FERROVIAIRE NOUVELLE}

Comment opérer la transition notionnelle d'une sensibilité propre à chaque chef d'entreprise vers des sensibilités d'ordre collectif et varié (Thévenot, 2006) à l'échelle des territoires? Assurément, le

10. Seuls deux dirigeants de services supérieurs aux entreprises ont indiqué conduire une stratégie dépendante de manière significative de la LGV. Il s'agit pour l'un d'une relocalisation interne à l'agglomération, à proximité de la gare, et pour l'autre de l'ouverture d'un établissement en Bretagne. choix d'une enquête qualitative par entretien individuel se prête difficilement à la production d'un discours collectif, concerté ou consensuel. Dans notre recherche, l'émergence d'une sensibilité synthétique à l'échelon de l'aire urbaine procède toutefois d'une agrégation de discours convergents. Ces expressions d'acteurs, confrontées par des données statistiques locales, mettent en exergue des informations, des signes ou des enjeux manifestes d'évolution des dynamiques territoriales (Béfort et Nieddu, 2017). Si la diversité et le volume d'informations font la richesse de l'étude, l'interprétation de 179 discours singuliers présente le risque de se voir dépasser par la matière accumulée et la difficulté attenante d'en extraire un "dénominateur commun ». Indubitablement, pareille enquête n'est pas sans biais. Elle demeure en partie suspendue à la représentativité de l'échantillon d'entrepreneurs ayant bien voulu y participer.

Pour autant, il ne s'agit pas de donner une image simplifiée et caricaturale devant une double complexité, celle de dynamiques urbaines lentes et multifactorielles d'une part et, d'autre part, celle de la projection délicate de la réflexion des dirigeants sur les territoires. Car les discours ne sont ni unanimes, ni exaltés, ni même réellement affirmés dès avant la mise en service de l'infrastructure nouvelle, et ce surtout lorsqu'on évoque l'incidence de la LGV sur les relations internes au Grand Ouest et non seulement les liens radiaux avec l'île-de-France. Néanmoins, alors que les travaux empiriques conduits sur les dynamiques productives territorialisées prennent généralement la forme de monographies (Garnier, 2005), le choix de la forme typologique pour retranscrire une vision spatialisée du sentiment des entreprises autorise l'appréhension simultanée des convergences et divergences.

L'appréhension de la LGV par les 179 responsables d'entreprise sollicités permet ainsi d'esquisser une géographie de l'évolution potentielle des treize territoires de l'étude au regard des thèmes présentés supra (figure 1). Si chacun présente des caractéristiques et des enjeux propres, il est cependant possible d'identifier trois dynamiques territoriales distinctes : celle de la pointe bretonne, celle d'un système rennais en construction et celle de l'axe ligérien, du Mans à Nantes (figure 3 - planche X). La typologie suivante associe plusieurs portraits détaillés de territoire et s'inspire allégrement des 
discours tenus par les dirigeants d'entreprise en intégrant des citations d'entretien.

\section{Les changements non discriminants de la pointe bretonne (Brest, Quimper, Lorient)}

Bien qu'ils bénéficient d'une importante réduction de la distance-temps sur un aller-retour pour Paris, Brest, Lorient et Quimper ne restent pas moins très dépendants de l'offre aéroportuaire locale. Aussi, les entreprises de ces territoires n'anticipent pas de changer leurs pratiques de mobilité ou de réorganisation spatiale. La distance-temps ferroviaire avec Paris continue d'être considérée comme pénalisante. «On sait qu'on est au bout du monde [...]. Il y a ceux qui arrivent à capter les opportunités et ceux qui ont appris à s'en passer », résume un grand nom de l'économie cornouaillaise. Seule l'attractivité nouvelle conférée par l'accessibilité du Grand Ouest en général peut avoir quelques effets indirects sur les conditions de recrutement de certains profils qualifiés. Dans cette optique, plusieurs entreprises finistériennes affichent un réel intérêt pour l'amélioration de l'accessibilité ferroviaire en direction de Rennes.

\section{Lorient : La pertinence accrue du TGV conduira-t-elle à une concurrence modale aiguë?}

Avec un temps de trajet moyen avoisinant les trois heures pour Paris, Lorient est au point de rupture de cette classification. Les enjeux d'accessibilité de la troisième agglomération bretonne renvoient plus qu'ailleurs au choix modal entre le TGV et l'avion. Il semble en effet que le fameux « seuil des trois heures », qui n'a à l'origine qu'un fondement statistique, soit révélateur de certaines évolutions potentielles dans les pratiques de déplacement des entreprises lorientaises et de leurs partenaires.

Nombreux sont les dirigeants à espérer un jeu concurrentiel plus agressif entre offres aérienne et ferroviaire qui puisse conduire à une diminution du prix des billets. Elargir autrement dit les opportunités dès l'instant où "l'avion et le train deviendront à peu près équivalents en termes d'attractivité ", même si " pour le business cela ne va pas changer grand- chose ». Car en réalité, l'appréhension de la LGV par les dirigeants lorientais, comme finistériens, est passablement circonscrite aux enjeux du choix modal et n'atteint que rarement des dimensions stratégiques de l'activité.

\section{Brest et Quimper : la LGV BPL ne remettra pas fondamentalement en cause l'hégémonie de l'avion}

Brest et Quimper bénéficieront à la mise en service de la LGV BPL d'un gain de temps moyenne de plus de quarante-cinq minutes et de deux allersretours supplémentaires vers Paris. Pour autant, et en toute logique, parler de déplacements en TGV d'une durée moyenne de $3 \mathrm{~h} 42$ entre Brest et Paris apparaît incongru pour beaucoup de chefs d'entreprise.

Impossible de ne parler que de TGV quand l'avion constitue le mode de transport privilégié et occupe à ce point les esprits. Pour beaucoup d'entreprises interrogées les temps de parcours en train resteront rédhibitoires en 2017, et seul l'aérien est en mesure de pouvoir satisfaire leurs besoins de déplacement. "On ne se sent pas concernés par le ferroviaire» relève d'emblée l'un des responsables interrogés. Si la LGV BPL contribue à " passer du stade de la préhistoire ferroviaire au semi-contemporain ", elle ne peut répondre ni aux besoins de mobilité croissants vers les grandes métropoles nationales, ni aux attentes de préacheminement vers les aéroports franciliens, ni même aux besoins vitaux de maintien des centres de décision en Finistère exprimés par les fleurons de l'économie locale.

Alors que le prix de l'avion à Brest est souvent jugé " effrayant et de plus en plus pesant ", l'accessibilité accrue de Rennes en 2017 est perçue par certains acteurs brestois comme une entrave supplémentaire à leur compétitivité. Face aux contrariétés liées à la liaison aérienne avec la région parisienne, quelques acteurs finistériens trouvent une alternative non pas dans le TGV mais dans des projets plus ou moins aboutis d'aviation légère privée et mutualisée. Certains chefs de file de l'économie brestoise sont de ce point de vue catégoriques : «il n'y a pas d'autres solutions que l'aérien, il ne faut pas se leurrer». 


\section{L'intégration relative au système métro- politain rennais (Rennes, Saint-Malo, Saint-Brieuc, Vannes, Vitré, Laval)}

Depuis l'été 2017, Rennes n'est plus qu'à 1 h 39 en moyenne de Paris. Parmi les apports de la LGV BPL pressentis et mentionnés par les dirigeants, la faculté supplémentaire à attirer des ressources hautement qualifiées s'impose. C'est d'ailleurs ce qui fait la consistance de ce groupe comprenant des villes moyennes dans l'orbite du hub ferroviaire rennais : Saint-Malo, Saint-Brieuc, Vannes, Vitré, Laval. Au-delà de leur propre attractivité résidentielle et productive, ces agglomérations entendent bénéficier encore davantage de la dynamique métropolitaine de la capitale bretonne (AUDIAR, 2016).

Vitré espère ainsi que Rennes puisse attirer et retenir d'autant plus facilement ces ressources rares qui lui manquent et qui, pour la plupart sont originaires de la région Île-de-France. Économiquement, l'agglomération a su dès les années 1950 faire valoir sa localisation « aux portes de la Bretagne » ainsi que sa proximité avec le pôle scientifique et industriel de Rennes ${ }^{11}$. Depuis les années 2000, la périurbanisation grandissante de la capitale bretonne participe à l'imbrication croissante des bassins d'emploi vitréen et rennais. Parmi les nouveaux habitants de Vitré, nombreux sont ceux qui travaillent à Rennes, et à l'inverse, l'un des dirigeants rencontrés indiquait intégrer dans ses effectifs plus d'un tiers de collaborateurs habitant le centre de l'agglomération rennaise.

Cette situation prévaut également à Saint-Brieuc, mais avec une acuité moins forte. L'agglomération briochine est surement de celles qui bénéficient le plus des gains offerts par la LGV BPL, en termes de fréquence et de temps de trajet avec Paris. En intégrant le seuil psychologique des villes à un peu plus de deux heures de la capitale, les entreprises locales sont bien conscientes de la souplesse apportée par l'infrastructure nouvelle en ce qui concerne les allers-retours dans la journée et la visite des partenaires. Elles se préparent en outre à saisir les opportunités de la capitale bretonne en termes d'investis-

11. Petite ville à l'échelle des 13 territoires étudiés (un peu plus de 17100 habitants en 2011), Vitré est pourtant la première agglomération du panel en termes de croissance démographique entre 1982 et 2011 (Insee). Sa zone d'emploi, la plus industrielle de Bretagne, tire son dynamisme d'une position privilégiée sur l'axe Rennes-Paris (Baudequin et Maillochon, 2013; Baudelle et al., 2015). sement, de compétences, de marché et également d'infrastructure performante. La gare de Rennes, premier hub ferroviaire de la Grande vitesse dans le Grand Ouest, sera sans doute davantage utilisée par les usagers professionnels briochins, comme par ceux de Vannes ou de Saint-Malo.

Dans ce groupe de villes dont les acteurs économiques joueront de plus en plus la carte rennaise, on peut effectivement ranger les deux agglomérations de Vannes et de Saint-Malo. Cependant, certains acteurs économiques de ces deux dernières villes, dont les activités sont fortement orientées sur l'international, ont tenu à souligner l'indépendance de leur fonctionnement à l'égard du hub rennais. Ils ont néanmoins exprimé leur satisfaction quant à la nouvelle géographie induite par l'arrivée de la grande vitesse ferroviaire en Bretagne, en particulier pour aider à l'installation d'actifs franciliens hautement qualifiés dans les secteurs de niche comme le nautisme à Vannes ou la valorisation des ressources maritimes à Saint-Malo. Dans un environnement appréciable, avec de grands fleurons industriels, ces villes dont l'image est davantage touristique qu'économique, voient leurs situations territoriales confortées à la fois par la nouvelle accessibilité qu'offre la LGV BPL et par le développement, à proximité immédiate, du pôle métropolitain rennais (ainsi que nantais pour Vannes). En quête d'une plus grande attractivité à l'égard notamment des profils hautement qualifiés, les activités qui s'y développent ou qui souhaitent s'y localiser pourront mieux faire connaître leurs savoirfaire en jouant sur l'effet notoriété d'un littoral à deux heures trente de Paris. Elles devraient profiter à la fois des ressources et des opportunités de la métropole bretonne et d'une alternative séduisante d'implantation économique sur les littoraux désormais parmi les plus proches de la capitale.

\section{Laval : Confortation des choix économiques et enjeux de coopération avec Rennes}

Enfin, l'agglomération lavalloise présente une posture territoriale particulièrement intéressante, qui apparait en effet révélatrice de la construction progressive d'un système interurbain local impulsé par la capitale bretonne. La mise en service de la LGV BPL offre ainsi aux dirigeants d'entreprises locales et aux élus l'occasion d'exprimer leur vision de l'avenir de leur territoire. Depuis le 
Moyen Âge, Laval tire profit de sa position géographique sur la route de Paris à Brest, aux frontières de la Bretagne, de la Normandie et de l'Anjou. Les infrastructures de transports successives, aux premiers rangs desquelles l'autoroute A81 et la ligne TGV, ont conforté cette localisation privilégiée par des liaisons radiales avec la capitale. Dès l'été 2017, la mise en service de la LGV BPL a placé la préfecture de la Mayenne à 1 h 25 en moyenne de Paris-Montparnasse. Mais les enjeux ne s'arrêtent pas aux liaisons avec Paris puisque les temps de parcours diminuent également avec Rennes et Le Mans, ainsi que vers Angers et Nantes via la Virgule de Sablé-sur-Sarthe. Cette évolution cumulée de nombreuses accessibilités ferroviaires requiert ainsi de l'agglomération lavalloise qu'elle s'interroge sur la place qu'elle entend défendre dans le système territorial régional et national. Économiquement orientée vers Rennes plus que vers Le Mans ou les autres agglomérations de sa région administrative, la réflexion n'est pas aisée.

Fin connaisseurs du territoire, les dirigeants lavallois sont conscients de l'opportunité engendrée par la LGV BPL, mais également des faiblesses locales qui limiteraient les changements bénéfiques sur le tissu économique. Les discours convergent vers la nécessité d'améliorer la visibilité de l'agglomération, de donner à la ville des atouts pour exploiter la plus grande accessibilité avec Paris. "Je ne suis pas sûr que la LGV change grand-chose depuis Paris, car il y a surtout l'image de la ville. [...] Sans avoir mis un pied à Laval c'est sûr que ce n'est pas évident, il faut embrayer ce cercle vertueux. » Un brin provocateur, un grand acteur économique mayennais qui ne tarit pourtant pas d'éloge sur la qualité de vie locale souligne que "personne ne vient à Laval, c'est la pire image de ville que je connaisse ».

Face à cette problématique impérieuse du recrutement, de nombreux chefs d'entreprise mayennais identifient pourtant dans les gains de temps à l'échelle régionale un axe d'amélioration prometteur. « Il sera plus facile de convaincre un parisien qui a un projet dans le Grand Ouest, soit de venir s'installer à Laval, soit, et c'est là la principale amélioration, en lui proposant de s'installer à Rennes ». Aujourd'hui déjà, les relations pendulaires entre les deux villes sont relativement intenses, d'autant plus si l'on considère le fait que ces déplacements quotidiens sont transrégionaux. L'agglomération lavalloise attire davantage de navet- teurs en provenance de Rennes ou du Mans qu'elle n'en voit partir actuellement vers ces mêmes pôles urbains. Les grands industriels de Laval, au premier rang desquels le géant mondial du lait, présentent souvent une proportion significative voire majoritaire de collaborateurs habitant la région rennaise. Et il n'est pas improbable que cette dynamique aille en s'accentuant avec la mise en service de la LGV BPL.

En effet, considérons un ménage dont l'un des deux actifs travaille à Laval, l'autre à Rennes. Si le gain de temps est logiquement le même que l'on prenne le TGV dans un sens ou dans l'autre, la structure de l'offre ferroviaire à l'été 2017 autorise plus facilement des mobilités domicile-travail de Rennes vers Laval que l'inverse. Depuis Rennes, seize trains quotidiens sont compatibles avec l'agenda d'un navetteur travaillant à Laval, dont sept permettent un trajet inférieur à la demi-heure. En comparaison, un navetteur habitant Laval et travaillant à Rennes ne dispose que de neuf trains quotidiens susceptibles de convenir à sa pendularité, et seul un TGV permet d'effectuer la liaison en moins d'une demi-heure.

Plusieurs acteurs économiques ne s'y trompent pas : "Laval doit pouvoir être en mesure de jouer davantage la carte de Rennes. " "Devenir la troisième couronne rennaise » iront jusqu'à dire certains. $\mathrm{Si}$ «le sujet de la relation avec Rennes est très important ", les liens nouveaux permis via la Virgule de Sablé-surSarthe ne sont néanmoins pas négligés. Le rapprochement avec Nantes est salué dans la perspective d'une cohésion régionale plus aboutie mais ne semble pas occasionner une ambition commerciale manifeste de la part des firmes lavalloises. Quant à la proximité nouvelle avec Angers, elle pourrait en théorie occasionner une hausse des flux domicile-travail entrants sur le territoire lavallois. Néanmoins, la fréquence de cinq allers-retours quotidiens reliant Angers et Laval via la Virgule de Sablé-sur-Sarthe apparaît très insuffisante pour bousculer drastiquement le paysage de la pendularité ferroviaire à l'échelle régionale.

\section{Les évolutions atones des agglomérations de l'axe ligérien (Le Mans, Sablé-sur- Sarthe, Angers, Nantes)}

À la différence de Laval, les entreprises rencontrées au Mans n'anticipent pas, ou de manière très impalpable, la proximité nouvelle avec Rennes dans 
leur développement stratégique, pas plus qu'elles n'intègrent davantage Laval. Bien que globalement rassurées par la nouvelle offre TGV, la perte perçue d'un avantage comparatif vis-à-vis de la capitale bretonne, jugée plus attractive et dynamique, émerge des discours. "Si demain la banlieue de Paris c'est Rennes, que va devenir Le Mans? " s'interroge l'un des dirigeants. La montée en puissance de Rennes interpelle les acteurs économiques manceaux qui regardent avec un intérêt mêlé d'une certaine inquiétude la dynamique d'EuroRennes et son ambition de devenir, à l'image de la métropole sarthoise, une nouvelle plateforme d'activités alternative à une heure trente de l'Île-de-France. Néanmoins, il est frappant de constater que cette concurrence territoriale, brandie comme une vague menace, n'est jamais réellement explicitée. Il est bien difficile pour un chef d'entreprise manceau d'exprimer les modalités et enjeux concrets de la concurrence accrue de Rennes pour son activité. Car en réalité ce sentiment de perte relative n'est que la «face émotive » du discours des acteurs manceaux. Le véritable cheval de bataille est autre : préserver cet outil de travail stratégique que constitue le TGV depuis la mise en service de la LGV Atlantique en 1989.

À Sablé-sur-Sarthe, les positions semblent plus opportunistes que dans le reste du groupe. Et pour cause, le riche tissu économique local, porté notamment par de grandes entreprises agroalimentaires, se révèle passablement déséquilibré. Il présente certaines faiblesses structurelles mentionnées par les dirigeants rencontrés, parmi lesquelles figurent la difficulté à recruter des cadres et le manque d'attractivité résidentielle. Alors que la LGV BPL n’apporte qu'un gain insignifiant pour l'activité des entreprises saboliennes, la création de la Liaison rapide par la Virgule de Sablé-sur-Sarthe est perçue par certains comme une ouverture potentielle vers le vivier d'actifs qualifiés habitant Laval. L'une des entreprises rencontrées, qui a fait le choix mûrement réfléchi de s'installer à proximité de la gare, affiche d'ailleurs dans ses récentes offres d'emploi cette proximité nouvelle avec la préfecture de la Mayenne. Si le potentiel d'actifs qualifiés en provenance de Laval n'est pas comparable aux viviers manceaux et angevins, l'accessibilité accrue permettrait néanmoins d'assoir la place de Sablé-sur-Sarthe dans le système territorial régional. "Le problème de Sablé, c'est qu'elle est à la frontière des départements, comme noyée dans sa région. En s'ouvrant vers Laval et la Bretagne, on place le territoire au niveau régional ». Mais si la liaison ferroviaire nouvelle n'est qu'un support aux déplacements pendulaires entrants et ne s'accompagne pas d'activités commerciales renforcées pour les entreprises locales, n'y-a-t-il pas un risque de voir ce territoire «péri-métropolitain » se replier toujours plus sur des dimensions industrielles que l'on sait sensibles aux aléas conjoncturels?

Enfin, plus à l'Ouest des Pays de la Loire, Angers et Nantes ne bénéficient également qu'à la marge de la réduction de la distance-temps avec la capitale. Aussi les acteurs économiques ne voient aucune raison de modifier leurs pratiques de mobilité, et l'évocation des changements d'accessibilité régionale ne suscite souvent aucune préoccupation. À Angers, le désintérêt se teinte d'inquiétude. En effet, l'agglomération angevine a connu depuis l'été 2017 une nouvelle perte qualitative et quantitative de sa desserte TGV radiale avec Paris-Montparnasse. De trente-et-un allers-retours TGV directs quotidiens entre Angers Saint-Laud et Paris-Montparnasse avant la refonte horaire, la capitale de l'Anjou passera à vingt-neuf allers-retours TGV directs quotidiens, dont dix sans arrêt intermédiaire contre seize actuellement. Par contre, la mise en service de la Liaison rapide offre de nouvelles perspectives vers Laval (cinquante-six minutes) et Rennes (une heure trente-six). Mais du point de vue des dirigeants rencontrés, cette proximité nouvelle par le fer n'apparaît pas déterminante pour l'activité à court ou moyen terme. La voiture reste le mode privilégié à l'échelle régionale et les relations avec Laval et Rennes sont actuellement relativement faibles, même dans des secteurs a priori convergents comme les télécommunications et l'édition logicielle.

\section{Conclusion}

Les expériences passées convergent vers le fait que les grands projets d'infrastructure de transport ferroviaire sont un support privilégié d'action pour les acteurs institutionnels des territoires concernés (Delage, 2013). En revanche, les connaissances empiriques de la sensibilité ex ante des entreprises sont particulièrement modestes. L'analyse des entretiens conduits auprès de 179 responsables d'entreprise du Grand Ouest et d'Île-de-France fait apparaître des positions bien plus mesurées que les acteurs publics 
sur l'incidence anticipée de la mise en service de la LGV BPL. Les investissements de préfiguration et les anticipations stratégiques sont particulièrement limités. Si les dirigeants reconnaissent dans les territoires de Rennes, Laval, Saint-Malo, Saint-Brieuc, Vannes, Vitré, Lorient, voire Sablé-sur-Sarthe, l'effet d'aubaine de la LGV BPL, cette dernière n'est guère réfléchie comme l'instigatrice de changements majeurs dans les conditions de l'activité. Autrement dit, leur sensibilité est bien davantage la résultante de formes d'émotivité que de la sphère stratégique et organisationnelle dès avant la mise en service. Cela étant, l'acuité de l'appréhension varie selon plusieurs caractéristiques propres à la firme (étendue de marché, relations siège-établissements...) ou extérieures à l'organisation (secteur d'activité). La dynamique actuelle des territoires influe en outre largement sur l'appréhension de l'accessibilité accrue par les chefs d'entreprise. À travers une lecture géographique des interrelations économiques en cours et projetées, ce travail a esquissé une typologie de la sensibilité des entreprises pour les 13 agglomérations étudiées. En congruence avec la mise en service de la LGV BPL, des dynamiques communes semblent notamment confortées entre Rennes et les villes moyennes alentours, préfigurant éventuellement à l'avenir un système métropolitain institutionnalisé et structuré.

\section{Remerciements}

Nous témoignons de notre profonde gratitude à chacun des 179 responsables d'entreprise rencontrés, sans qui cette étude ne serait pas. Que les relecteurs anonymes de cet article soient également remerciés.

\section{Bibliographie}

Albalate D., Bel G., 2012. High-speed rail: lessons for policy makers from experiences abroad, Public Administration Review, no 72 (3), p. 336-349.

AUDIAR, 2016. Rennes Métropole et les territoires bretons, une relation profitable à l'ensemble des partenaires, Rennes, $64 \mathrm{p}$.

Baudelle G., Fache J. (dir.), 2015. Les mutations des systèmes productifs en France, Rennes, PUR, 374 p.

Baudequin I., Maillochon A., 2013. Le tissu industriel des Pays de Fougères et Vitré : une armature solide confrontée à de nouveaux défis, Insee Bretagne - Octant analyse, $\mathrm{n}^{\circ}$ 42, décembre 2013.
Bavoux J., Piquant M., 2000. Les réseaux de transport entre Paris et Lyon : compétition ou coopération?, Flux, n 39-40, p. 30-39.

Bazin S., Beckerich C., Delaplace M., 2006. Analyse prospective des impacts de la Ligne Grande Vitesse Est-Européenne dans l'agglomération rémoise et en région ChampagneArdenne. Rapport final remis à la région ChampagneArdenne, 555 p.

Bazin S., Beckerich C., Delaplace M., Blanquart C., VanDENBossche L., 2010. Lignes ferroviaires à grande vitesse et dynamiques locales : une analyse comparée de la littérature. Comité National de Géographie. Transport et développement des territoires, Le Havre, France, 21 p.

BÉfort N., Nieddu M., 2017. De la variété des ancrages territoriaux des firmes en chimie doublement verte, Géographie, économie, société, no 19 , p. 133-152.

BÉrion P., 1998. Analyser les mobilités et le rayonnement des villes pour révéler les effets territoriaux des grandes infrastructures de transport, Les cahiers scientifiques du transport, no 33, p. 109-127.

Bizeray N., Blanquart C., Burmeister A., Colletis-Wahl K., 1996. Infrastructures de transport et développement : de l'effet vers l'interaction. Transports, $n^{\circ} 377$, mai-juin, p. 202-208.

Blanquart C., Delaplace M., 2009. Innovations relationnelles, nouvelles offres de services et valorisation des nouvelles infrastructures de transport, Les cahiers scientifiques du transport, $\mathrm{n}^{\circ} 56$, p. 63-86.

Blanquart C., Joignaux G., Vaillant L., 2013. Infrastructure de transport et développement économique : quelles dynamiques d'appropriation par les acteurs productifs? L'exemple de la filière céréalière autour du canal SeineNord Europe, Revue d'Économie Régionale \& Urbaine, $\mathrm{n}^{\circ} 1$ (février), p. 119-138.

Bonnafous A., Plassard F., 1974. Les méthodologies usuelles de l'étude des effets structurants de l'offre de transport, Revue économique, $\mathrm{n}^{\circ} 25$ (2), p. 208-232.

Bouba-Olga O., Ferru M., Guimond B., 2012. Organisation des activités et dynamiques territoriales : éléments d'analyse et application aux bassins de Cognac et de Châtellerault, Revue d'Économie Régionale E Urbaine, $\mathrm{n}^{\circ} 2$ (avril), p. 173-191.

Buisson M.-A., 1986. Effets indirects du TGV et transformations du tertiaire supérieur en Rhône-Alpes, Études et Recherches. LET, Lyon, 87 p.

Carrouet G., 2013. Du TGV Rhin-Rhône au "Territoire » Rhin-Rhône : réticularité, mobilité et territorialité dans un espace intermédiaire. Thèse de doctorat en géographie, Université de Bourgogne, 527 p.

CGSP, 2013. L'évaluation socio-économique des investissements publics, rapport de la commission présidée par Émile Quinet, Commissariat général à la Stratégie et à la prospective, septembre 2013.

Chevallier J., 1990. Le Mans à l'arrivée du TGV Atlantique, Rapport d'étude de l'OEST, décembre, 187 p.

Combes P.-P., Mayer T., Thisse J.-F., 2006. Économie géographique : l'intégration des régions et des nations, Paris, Economica, coll. «Corpus Économie », 397 p. 
Crozet Y., 2013. Performances économiques des régions françaises et investissements en infrastructures de transport. Support de cours à l'IEP de Lyon, 3 p. (non publié).

Delage A., 2013. La gare, assurance métropolitaine de la ville post-industrielle. Le retournement de valeur dans les projets urbains de quartiers de gare, à Saint-Étienne Châteaucreux et Liège Guillemins (Belgique). Thèse de doctorat en géographie, Université Lumière Lyon 2, 542 p.

Delaplace M., Bazin S., Beckerich C., Blanquart C., 2013. Les enjeux et opportunités de la Grande Vitesse Ferroviaire en termes de développement local et de développement durable: une analyse fondée sur la production et l'appropriation des innovations de services. Rapport de recherche PREDIT, 188 p.

Dornbusch J., 1997. Nantes, sept ans après l'arrivée du TGV Atlantique. Notes de synthèse du SES, mai-juin, 4 p.

Facchinetti-Mannone V., 1995. L'impact régional du TGV sud-est. Thèse de doctorat en géographie, Université AixMarseille 1, 567 p.

Frémont A., 2014. Les effets structurants ou les raccourcis de l'explication géographique, in Beaucire F., Delaplace M., Ninot O., Bretagnolle A., Pumain D., Offner J.-M. (dir.), Les effets structurants des infrastructures de transport. Espace géographique, no 43 (1), p. 65-67.

GAC G., 1990. Effets socio-économiques du TGV A au Mans: restructuration du centre, conséquence la plus immédiate, Notes de synthèse de l'OEST, février, 4 p.

Garnier J., 2005. Proximités lourdes, proximités légères : une trajectoire de l'appareil productif dans l'aire métropolitaine marseillaise, Géographie, économie, société, Vol. 7, p. 365-380.

Giuliani E., 2007. The selective nature of knowledge networks in clusters: evidence from the wine industry, Journal of Economic Geography, no 7 (2), p. 139-168.

KLein O., 1997. Le TGV-Atlantique et les évolutions de la mobilité : entre crise et concurrence, Les cahiers scientifiques du transport, $\mathrm{n}^{\circ} 32$, p. 57-83.

KLEIN O., 2001. Les horizons de la grande vitesse: Le TGV, une innovation lue à travers les mutations de son époque. Thèse de doctorat Economies et finances, Université Lumière Lyon 2, 331 p.

KLein O., 2005. Temporalités sociales et gestion de la distance à travers les moyens de transport rapides, in Montulet B., Hubert M., Jemelin C., Schmitz S. (eds.), Mobilités et temporalités, Facultés universitaires Saint-Louis, collection Travaux et Recherches, no 51 (2), p. 21-33.

Klein O., Million F., 2007. La GVF, le développement socioéconomique et l'aménagement des territoires: étude bibliographique et analyse des expériences passées. Rapport à la DDE de Charente, $174 \mathrm{p}$.

Lafourcade M., Thisse J.-F., 2009. New economic geography : the role of transport costs, in Palma A., De Lindsey R., Quinet E., Vickerman R. (dir.), Handbook in Transport Economics, Edward Elgar Publishing, London, 928 p.

LEE Y.S., 2007. A Study of the development and issues concerning High Speed Rail (HSR). Korea Railroad Research Institute and
Transport Studies Unit, University of Oxford, Working paper $\mathrm{n}^{\circ} 1020$, Oxford University Centre for the Environment, 19 p.

Martin R., Sunley P., 2007. Complexity thinking and evolutionary economic geography, Journal of Economic Geography, $\mathrm{n}^{\circ} 7$ (5), p. 573-601.

Mattmuller M., 2016. Analyse comparée des trajectoires des métropoles nationales. Rapport de stage de fin d'études, ENSAI, Université Bretagne Loire, 72 p. (non publié).

Menerault P., 2000. Appropriation régionale de la grande vitesse ferroviaire et rôle des régions dans la dynamique des réseaux: le cas du TGV en Wallonie et en Bretagne. Groupement Régional pour la Recherche dans les Transports, 105 p.

Mérenne-Schoumaker B., 1996. La localisation des services, collection Géographie d'aujourd'hui, Nathan, Paris, 1992 p.

Mérenne-Schoumaker B., 2011 . La localisation des industries. Enjeux et dynamiques, Rennes, Presses universitaires de Rennes, nouvelle éd., coll. « Didact Géographie », 255 p.

Mignerey P., 2012. Les effets territoriaux de la grande vitesse ferroviaire en France, La Documentation française, DATAR, $96 \mathrm{p}$.

NÈGRE R., 20I8. Sensibilité prospective des entreprises à l'amélioration de l'accessibilité ferroviaire: le cas de la ligne à grande vitesse Bretagne - Pays de la Loire. Thèse de doctorat en géographie, Université Rennes 2, 269 p. [publication à venir]

Offner J.-M., 1993. Les « effets structurants » du transport: mythe politique, mystification scientifique, L'espace géographique, $\mathrm{n}^{\circ} 22$ (3), p. 233-242.

Ollivro J., 1997. TGV et fonctions supérieures dans les régions Bretagne et Pays de la Loire. Rapport établi à l'initiative du ministère de l'Équipement, du Logement, des Transports et du Tourisme, Université de Rennes 2.

Paumier J.-Y., 1990. Nantes à l'arrivée du TGV Atlantique, Rapport d'étude de l'OEST, décembre, 163 p.

Perrat J., 2000. Dynamique des firmes et politiques de développement régional et local : éléments pour une lecture de la "géographie des interdépendances », in GiLLY J.-P., Torre A. (dir.), Dynamiques de Proximité, Paris, L'Harmattan, p. 101-121.

ThéVenot L., 2006. L'action au pluriel : sociologie des régimes d'engagement, série Politique et sociétés, La Découverte, Paris, $312 \mathrm{p}$.

Torre A., Rallet A., 2005. Proximity and localization, Regional Studies, no 39 (1), p. 47-60.

Ureña J.-M., Menerault P., Garmendia M., 2009. The highspeed rail challenge for big intermediate cities: A national, regional and local perspective, Cities, n² 26, p. 266-279.

Vickerman R.-W., 1991. Infrastructure and regional development, Pion Ltd, London, 209 p.

Vickerman R.-W., Ulied A., 2012. Indirect and wider economic impacts of high-speed rail, in De Rus, G. (ed.), Economic analysis of High Speed Rail in Europe, Fundacion BBVA, p. 91-107. 